\title{
The methanol maser ring G23.657-00.127 after 9 years
}

\author{
Anna Bartkiewicz* \\ Torun Centre for Astronomy, Faculty of Physics, Astronomy and Informatics, Nicolaus \\ Copernicus University, Poland \\ E-mail: annan@astro.umk.pl
}

\begin{abstract}
Alberto Sanna
Max-Planck-Institut fuer Radioastronomie, Auf dem Huegel 69, Bonn, Germany

E-mail: asanna@mpifr-bonn.mpg.de
\end{abstract}

\section{Marian Szymczak}

Torun Centre for Astronomy, Faculty of Physics, Astronomy and Informatics, Nicolaus Copernicus University, Poland

E-mail: msz@astro.umk.pl

\section{Luca Moscadelli}

Osservatorio Astrofisico di Arcetri, Firenze, Italy

E-mail: moscadarcetri.astro.it

\begin{abstract}
We report about a multi-epoch study of the $6.7 \mathrm{GHz}$ methanol masers toward the high-mass, young stellar object, G23.657-00.127. Its maser distribution shows a peculiar ring-like morphology that we re-observed after 8.5 years, in order to measure the internal kinematics of the ring. A preliminary result of the maser proper motion studies suggests a combination of an expansion and rotation along the ring. These results are at variance with the expectations for an infalling envelope, and support a scenario where the masing gas emerges at the base of the inner outflow.
\end{abstract}

12th European VLBI Network Symposium and Users Meeting

7-10 October 2014

Cagliari, Italy

\footnotetext{
*Speaker.
} 


\section{Introduction}

The methanol masers at $6.7 \mathrm{GHz}$ are indicators of an early stage of high-mass star-formation [6]. By means of VLBI observations, they enable us to study the neutral gas at milliarcsecond (mas) scales in close vicinity of forming massive stars. The spatial distributions of methanol maser spots are known to show different morphologies, from compact to arc-like or irregular structures (e.g. [7], [10], [16]). Using the $\mathrm{EVN}^{1}, 31$ methanol sources towards the Galactic plane were imaged with a few mJy sensitivity and a few mas angular resolution [2]. Among that sample one source, G23.657-00.127, showed nearly-circular symmetric structure and became a prototype of the (so called) ring-like morphology of methanol masers [2]. Such symmetric structures suggested that: i) there is a central object(s) at the origin of the ring; ii) in these sources, methanol masers are likely associated with gas in a circumstellar disc or torus. However, by applying a simple model for a rotating and expanding thin disc (e.g. [15]), we showed that such ring-like structures are not dominated by rotation [2]. Instead expanding or infalling motions seem to dominate the maser kinematics, suggesting that the methanol masers are rather related to outflows and/or the interaction zone between the outflow and the accretion disc (e.g. [14]).

In order to address the question, what kind of kinematics are methanol rings tracing?, we started a systematic campaign of proper motion measurements of such ring-like structures as the most direct investigation of their nature. VLBI observations are a powerful tool to trace the 3D kinematics of the masing gas over time baselines of a few years (e.g. [8], [9]). Rotation combined with expansion around the jet/outflow axis, or the infall of a molecular envelope, were recently reported for some HMSFRs (e.g. [11], [12], [4]).

Here, we present preliminary results of a systematic, proper motion study of the maser kinematics in G23.657-00.127, as part of a large-project which targets the whole sample of methanol maser rings reported by [2]. Up to now, we have collected two epochs of EVN observations separated by 8.5 years. Such time baseline is appropriate to detect even small shifts in the position of single maser spots, as expected for maser velocities as small as a few $\mathrm{km} \mathrm{s}^{-1}$ at the distance of the source of $3.19 \mathrm{kpc}$ [1]. A third epoch of EVN observations toward G23.657-00.127 is expected in 2015 (EVN session I).

\section{Observations}

The first epoch data were observed in the EN003 project taken on 11 November 2004 that was reported by [2]. The second epoch data were observed on 2 March 2013 (project code EB052) using the EVN with the following antennas: Jodrell Bank, Effelsberg, Medicina, Noto, Onsala, Torun, Westerbork and Yebes. The observations were phase-referenced using J1825-0737 with a switching cycle of $175 \mathrm{~s}+95 \mathrm{~s}$ (maser+phase-calibrator) giving a total on-source time of $1.4 \mathrm{~h}$. Using the EVN Mk IV Data Processor at JIVE, we set a spectral resolution on the maser lines of $0.1 \mathrm{~km} \mathrm{~s}^{-1}$, across a bandwidth of $2 \mathrm{MHz}$ divided into 1024 spectral channels. In order to increase the signal-to-noise ratio on the phase-reference source, we used eight BBCs per polarization for a second correlator pass with 128 channels per BBC (each $2 \mathrm{MHz}$ wide).

\footnotetext{
${ }^{1}$ The European VLBI Network is a joint facility of European, Chinese, South African and other astronomy institutes funded by their national research councils.
} 


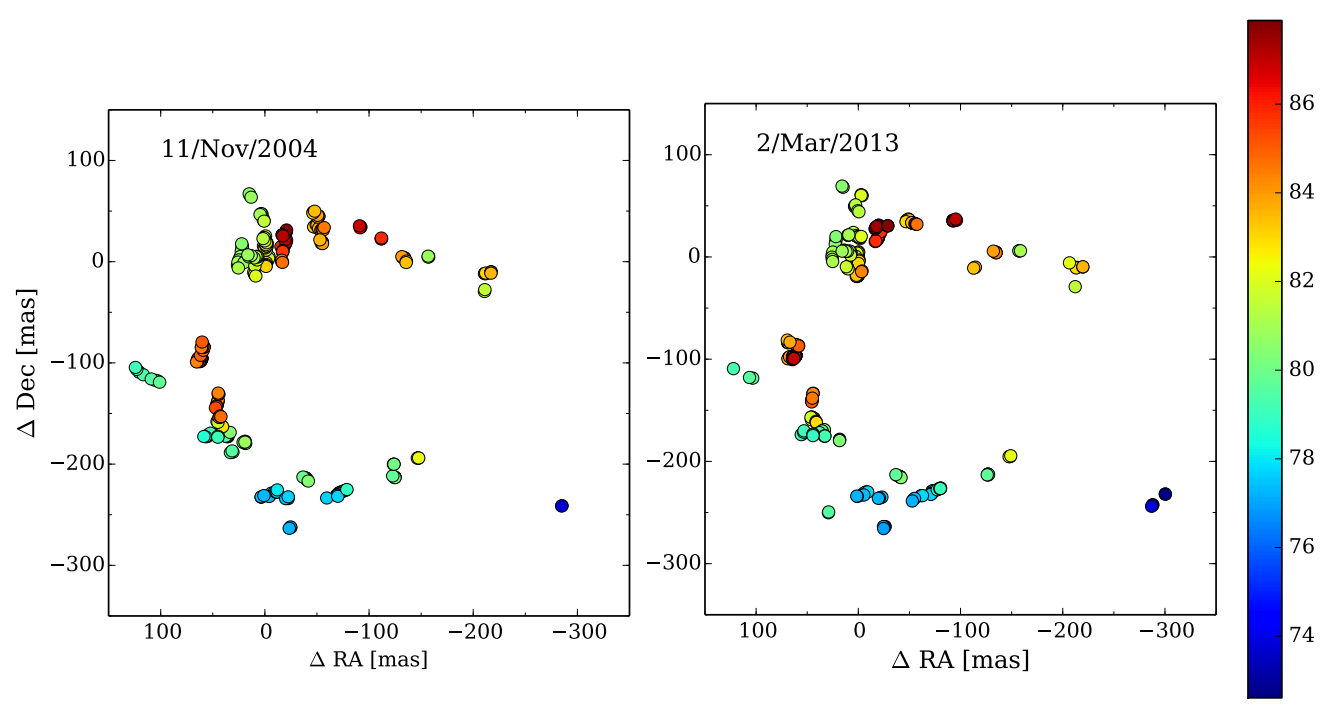

Figure 1: The $6.7 \mathrm{GHz}$ methanol maser spot distributions towards G23.657-00.127 obtained using the EVN at two different epochs, separated by 8.5 years.

The data reduction was carried out in AIPS, employing the standard procedures for spectral line observations. Phase calibration was performed on the strongest maser channel at a $\mathrm{V}_{\mathrm{LSR}}$ of $82.5 \mathrm{~km} \mathrm{~s}^{-1}$. Finally, we searched for maser emission within a radius of about $1 \operatorname{arcsec}$ (or about $3200 \mathrm{AU}$ ) around the strongest maser spot, by using the task SAD of AIPS and a cutoff of $7 \sigma$ on each channel map.

\section{Results and Discussion}

In total, we registered 325 methanol maser spots towards G23.657-00.127 in the second epoch data; the brightest spot was $2.2 \mathrm{Jy}^{\text {beam }^{-1}}$ while the weakest one was $0.026 \mathrm{Jy} \mathrm{beam}^{-1}$. Fig. 1 compares the spot distributions from 2004 and 2013 by aligning the brightest spot at each epoch, at a $\mathrm{V}_{\mathrm{LSR}}$ of $82.5 \mathrm{~km} \mathrm{~s}^{-1}$, with the $(0,0)$ point. The overall structure clearly persisted for 8.5 years, covering both the same $\mathrm{V}_{\mathrm{LSR}}$ and brightness ranges. The centres of best fitted ellipses to the data from both epochs (excluding the blueshifted spots at $73.6 \mathrm{~km} \mathrm{~s}^{-1}$ ) differ by 0.2 mas and 0.1 mas in RA and Dec, respectively. Their major and minor semi-axes were 129.7 mas, 135.1 mas (2004) and 130.7 mas and 135.3 mas (2013), respectively. The position angles of the major axes were $66^{\circ}$ (2004) and $63^{\circ}$ (2013) (north to east), respectively.

In order to derive the internal kinematics of the methanol maser ring, we firstly aligned the centres of the best fitted ellipses. This approach removes any bulk motion of the ring in the plane of the sky. Next, we identified 198 pairs of spots that appeared at both epochs at the same LSR velocity (within two spectral channels) and at the same relative position on the sky (within 10 mas). These pairs were distributed along the whole ring and belonged to 34 maser groups (i.e. features) clearly separated each one from the other. We built up the proper motion vector taking the average of the positional shifts between corresponding pairs in each feature. The final plot is presented in Fig. 2, where we show the barycentre of each feature (dots) and its proper motion vector (arrows). 


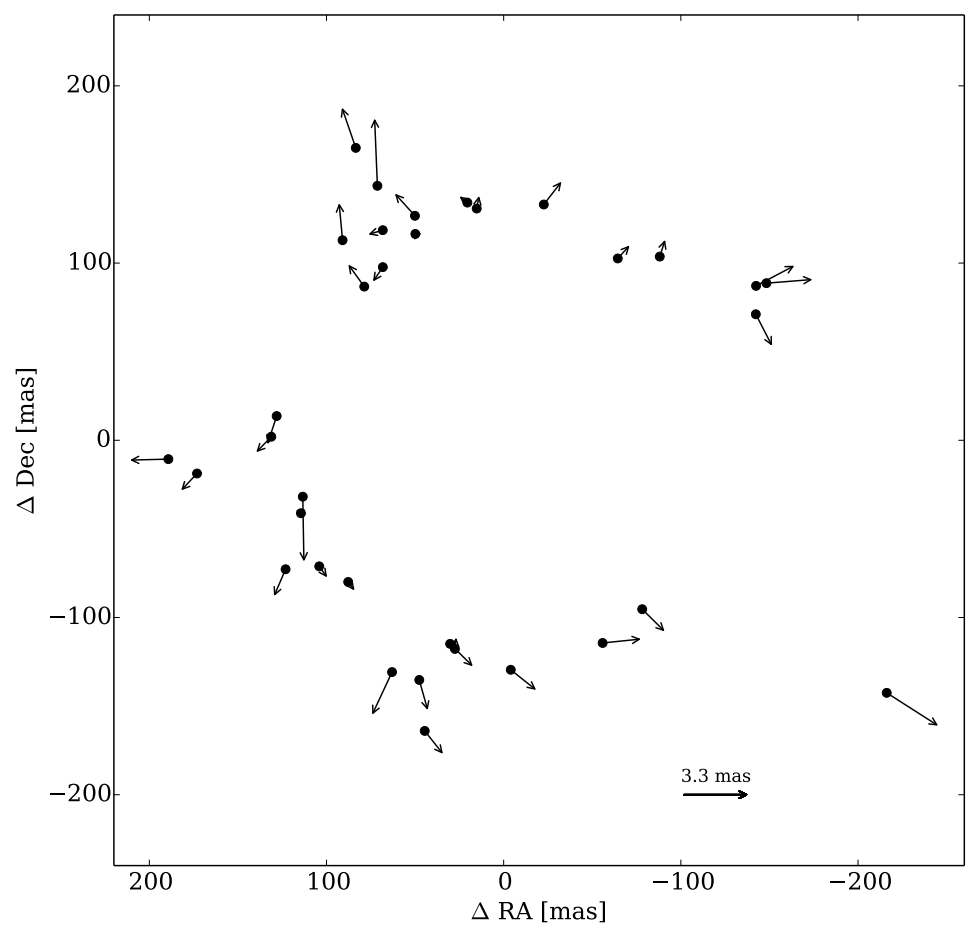

Figure 2: Proper motion vectors of the $6.7 \mathrm{GHz}$ methanol maser features in G23.657-00.127 in the period from 2004 to 2013 . The $(0,0)$ point corresponds to the centre of the best fitted ellipse to the data from 2013.

This result clearly shows that the maser kinematics in G23.657-00.127 cannot be explained by an infalling motion of the ring (e.g. [4], [13]).

Then, we compared for each feature the difference between its position vector (relative to the centre of the best fitted ellipse) and the direction of its proper motion vector. A coincidence between these two vectors would suggest radial motion (expansion or infall), while a difference of $90^{\circ}$ would suggest rotation. The mean value is $\theta=38^{\circ}$ with a standard deviation of $5^{\circ}$. A possible explanation would be a combination of rotation and expansion. We also checked the dependence of the magnitude of the proper motion vectors with the position of each feature along the ring. We do not note any particular direction where the motion would be either significantly greater or smaller (Fig. 3). The mean shift after 8.5 years is 1.53 mas with a standard deviation of 0.13 mas. At the source distance of $3.19 \mathrm{kpc}$ [1], this corresponds to $4.9 \mathrm{AU}$ and $2.7 \mathrm{~km} \mathrm{~s}^{-1}$. Taking the value of $\theta$ of $38^{\circ}$, as calculated above, one can derive the two components of the mean velocity that are a mean radial velocity of $1.7 \mathrm{~km} \mathrm{~s}^{-1}$ and a mean rotation velocity of $2.13 \mathrm{~km} \mathrm{~s}^{-1}$ as sine and cosine functions of $\theta$, respectively. These values are smaller than the best fitted parameters obtained with the first epoch only, by assuming a thin Keplerian disc model [2].

A similar trend was previously reported toward the HMSFR G23.01-0.41. In this source, $6.7 \mathrm{GHz}$ methanol masers emerge from the inner portion of a molecular toroid with velocities of a few $\mathrm{km} \mathrm{s}^{-1}$, tracing a combination of rotation around the outflow axis and expansion along this 


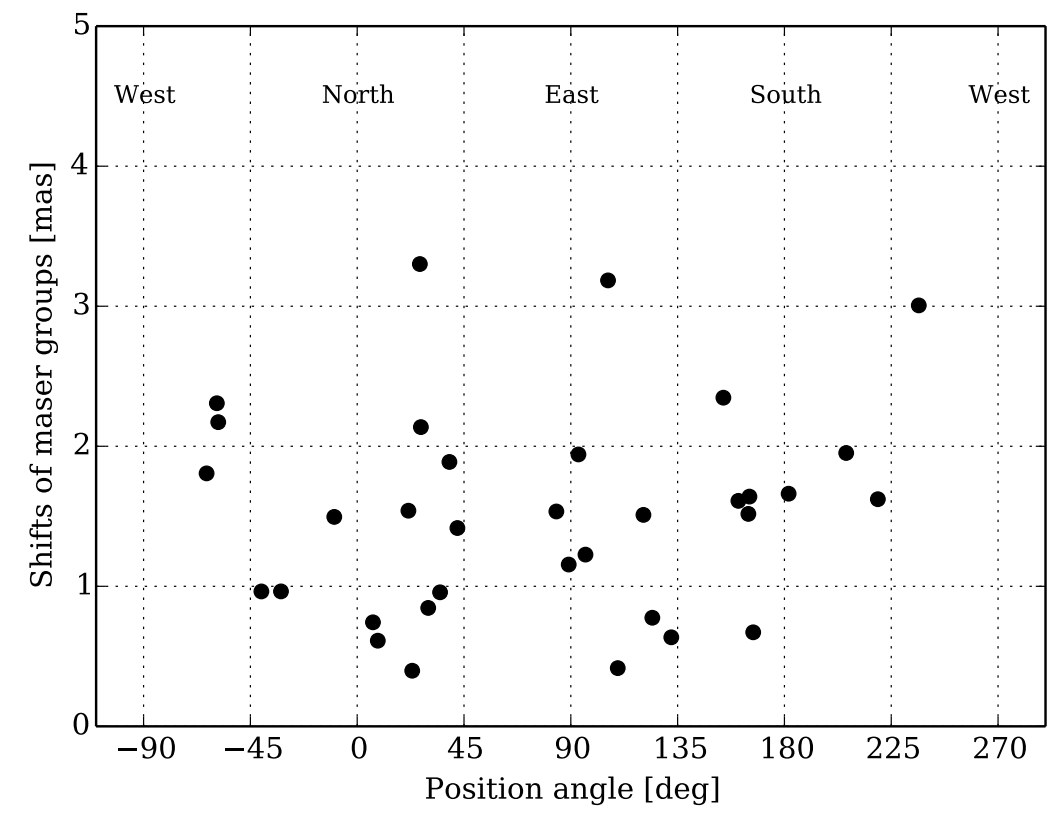

Figure 3: Position shift of maser features between 2004 and 2013 versus their position angle with respect to the centre of the best fitted ellipse.

axis [12].

Moreover, we also note that the northern features in G23.657-00.127 show the rotation components oriented clockwise, while the southern anticlockwise (Fig. 2). This change takes place at the position angle of ca. $50^{\circ}$ and might be related to the outflow since such direction points to the centre of a nearby infrared source located at the SW of the ring centre [3].

The third epoch of data will improve the accuracy of the proper motions and help to discriminate between these two scenarios described above.

\section{Acknowledgements}

We are grateful to dr. hab. Krzysztof Katarzynski for helping in the Python programming. AB and MS acknowledge support from the National Science Centre Poland through grant 2011/03/B/ST9/ 00627. This research is supported by the European Community Framework Programme 7, Advanced Radio Astronomy in Europe, grant agreement No. 283393.

\section{References}

[1] A. Bartkiewicz, A. Brunthaler, M. Szymczak, H.J. van Langevelde, M.J. Reid The nature of the methanol maser ring G23.657-00.127. I. The distance through trigonometric parallax measurements, A\&A, 2008, 490, 787-792

[2] A. Bartkiewicz, M. Szymczak, H.J. van Langevelde, A.M.S. Richards \& Y.M. Pihlström The diversity of methanol maser morphologies from VLBI observations, A\&A, 2009, 502, 155 
[3] J.M. De Buizer, A. Bartkiewicz, M. Szymczak Testing the Hypothesis that Methanol Maser Rings Trace Circumstellar Disks: High-resolution Near-infrared and Mid-infrared Imaging, ApJ, 2012, 754, 149, 1-15

[4] C. Goddi, L. Moscadelli, A .Sanna Infall and outflow within 400 AU from a high-mass protostar. 3D velocity fields from methanol and water masers in AFLG 5142, A\&A, 2011, 535, L8, 1-5

[5] N. Matsumoto, M. Honma, Y. Isono, et al., Astrometry of 6.7GHz Methanol Maser toward W3(OH) with Japanese VLBI Network, PASJ, 2011, 63, 6, 1345-1356

[6] K.M. Menten, The discovery of new, very strong and widespread interstellar methanol maser transition, ApJ, 1991, 380, L75-78

[7] V. Minier, R.S. Booth \& J.E. Conway, VLBI observations of 6.7 and $12.2 \mathrm{GHz}$ methanol masers toward high mass star-forming regions, A\&A, 2000, 362, 1093-1108

[8] L. Moscadelli, R. Cesaroni \& M.J. Rioja, Tracing the root of the bipolar jet in IRAS 20126+4104: VLBA observations of H2O masers, A\&A, 2000, 360, 663-670

[9] L. Moscadelli, R. Cesaroni \& M.J. Rioja, Water masers in the massive protostar IRAS 20126+4104: ejection and deceleration, A\&A, 2005, 438, 889-898

[10] C.J. Phillips, R.P. Norris, S.P. Ellingsen \& P.M. McCulloch, Methanol masers and their environment at high-resolution, MNRAS, 1998, 300, 1131-1157

[11] A .Sanna, L. Moscadelli, R. Cesaroni et al., VLBI study of maser kinematics in high-mass star-forming regions. I. G16.59-0.05, A\&A, 2010, 517, A71, 1-15

[12] A .Sanna, L. Moscadelli, R. Cesaroni et al., VLBI study of maser kinematics in high-mass star-forming regions. II. G23.01-0.41, A\&A, 2010, 517, A78, 1-19

[13] K. Sugiyama, K. Fujisawa, A. Doi et al., Rotating and infalling motion around the high-mass young stellar object Cepheus A-HW2 observed with the methanol maser at $6.7 \mathrm{GHz}$, A\&A, 2014, 562, A82, $1-13$

[14] K.J.E. Torstensson, H.J van Langevelde, W. Vlemmings \& S. Bourke, Dynamics of the 6.7 and 12.2 GHz methanol masers around Cepheus A HW2, A\&A, 2011, 526, A38, 1-9

[15] L. Uscanga, Y. Gómez, A.C. Raga, J. Cantó, G. Anglada, J.F. Gómez, J.M. Torrelles \& L.F. Miranda Kinematics of the $\mathrm{H}_{2} \mathrm{O}$ masers at the centre of the planetary nebula K3-35, MNRAS, 2008, 390, $1127-1132$

[16] A.J. Walsh, M.G. Burton, A.R. Hyland \& G. Robinson, Studies of ultracompact H II regions, MNRAS, 1998, 301, 640-698 